\title{
Experimental Studies on Relapse of Full-Term Neonatal Jaundice after Phototherapy
}

\author{
Li Da Mo ${ }^{1}$, Ling Yu Lu ${ }^{2}$ and Guo Sheng Su*3 \\ ${ }^{1}$ Deputy Chief Technician, The clinical laboratory of the Fourth people's Hospital, China \\ ${ }^{2}$ Deputy Director Technician, Director of Inspection Department, The clinical laboratory of the maternal and child care service centre, China \\ ${ }^{3}$ Deputy Chief Technician, Director of Inspection Department, The clinical laboratory of Orthopedic Hospital of Integrated Chinese and Western Medicine, \\ China
}

Received: June 04, 2018; Published: June 13, 2018

*Corresponding author: Su Guo Sheng, Deputy Chief Technician, Director of Inspection Department, Department of Clinical Laboratory of Orthopedic Hospital of Integrated Traditional Chinese and Western Medicine, Guigang City, Guangxi, China

\begin{abstract}
Objective: Learn about the status of laboratory testing relapse after neonatal jaundice phototherapy.

Methods: Sample of phototherapy full-term neonatal children with high blood bilirubin 222 cases, divided into 2 groups, according to the treatment result Relapse Group After phototherapy (31 cases), and NO Relapse Group After phototherapy (191 cases).Observing key laboratory test index of two groups such as G6PD, blood, blood routine, liver meritorious service etc.

Results: Before Phototherapy, the Hb of Relapse Group After Phototherapy (149.63 \pm 14.71$) \mathrm{g} / \mathrm{l}$ is obviously lower than these $(156.02 \pm 17.90)$ $\mathrm{g} / \mathrm{l}$ of NO Relapse After Phototherapy Group, $\mathrm{t}=2.169, \mathrm{P}=0.035$.After Phototherapy, the incidence of ABO hemolytic disease of newborn of Relapse Group is (16.13\%) significantly higher than those(4.71\%) of NO Relapse Grou , $t=5.857, \mathrm{P}=0.016$; And the difference between other indicators of the two groups of neonate has no statistical significance $(\mathrm{P}<0.050)$.Those indicators are AFP, ALT, AST, TBIL, HCT, MCHC and G6PD.

Conclusion: Relapse of full-term neonatal jaundice after phototherapy may be related to lower hemoglobin concentration and ABO hemolytic disease. In order to improve curative effect, these risk factors should be taken into consideration when making Follow-up plan after phototherapy.

Keywords: Neonate; Jaundice; Phototherapy; Laboratory Test Indicators

Abbreviations: AFP: Alpha Fetoprotein; ALT: Alanine Aminotransferase; AST: Aspartate Aminotransferase; TBil: Total Bilirubin; TBA: Total Bile Acid; G6PD: Glucose 6-Phosphatedehydrogenase
\end{abstract}

\section{Introduction}

Neonatal jaundice is the most common symptoms of newborns. High serum bilirubin to a certain extent can appear bilirubin encephalopathy that Causing harm to health. The most commonly treatment is blue light therapy (phototherapy) with distinct Curative effect, convenient and simple and less side effects. But a small number of newborn relapse after phototherapy whose Serum bilirubin increased to the point of high blood bilirubin. They need to re-treatment. At home and abroad have reported: it is a risk factor for high blood bilirubin that Neonatal jaundice recurrence after phototherapy [1,2]. Research on laboratory test indicator that relevant to neonatal jaundice relapsing after phototherapy has made great progress. But most research is based on one or two test indicators. On the contrary, this study by analyzing a variety of detection indicators aimed to explore what factors lead to neonatal jaundice apparent relapse after phototherapy .And relevant treatment measures be taken so that to ensure the newborn grow up healthily.

\section{Materials and Methods}

\section{The general information In January 2012 to December 2015}

There are 242 cases newborn children with high blood bilirubin receiving phototherapy (blue light treatment) in our hospital maternity ward. Considering some factors may affect the research results such as complications or physiological jaundice lasting too long, we exclude 8 cases whose mother with Acquired immunodeficiency disease and 12 cases that Premature babies or Low birth weight babies and take the remaining 222 cases as the research object which be divided into 2 groups, according 
to the treatment result: Relapse Group After phototherapy (31 cases), and NO Relapse Group After phototherapy (191 cases). The differences of below general information between 2 groups have no statistical significance $(\mathrm{P}>0.05)$. The general information are gender, gestational age, birth weight and maternal age, pregnancy time, production time, I score (including general reaction, the degree of mental state, hard swollen area, crying, vomiting, sputum chirp, cranial hematoma, breathing, heart, lung, liver and spleen enlargement), Jaundice time and phototherapy time. The compares of 2 groups Children mother common complication in the perinatal period has no Statistical significance as well $(\mathrm{P}>0.05)$. HBsAg positive, Gynecological inflammation, respiratory tract infection, TPHA positive, HCMV infection, infection of HSV-2, thyroid dysfunction, diabetes, hypertension, light-duty Mediterranean anemia and myoma of uterus.It is comparable, as below Tables 1 \& 2 shows. This research follows the process according with our ethical and moral ethics standards set by the management committee and approved by the committee. It implement after Signing Clinical study of informed consent with the guardian of the children.

Table 1: Comparison of the two groups' General data ( $x \notin 6$ ).

\begin{tabular}{|c|c|c|c|c|}
\hline Groups & $\begin{array}{c}\text { Relapse Group After } \\
\text { phototherapy(n=3) }\end{array}$ & $\begin{array}{c}\text { No Relapse Group After } \\
\text { phototherapy(n=191) }\end{array}$ & t/X2 & P \\
\hline Gender (male/female) & $18 / 13$ & $105 / 86$ & 0.103 & 0.749 \\
\hline Gestational age (week) & $38.87 \pm 1.31$ & $39.35 \pm 1.03$ & 1.956 & 0.058 \\
\hline Birth weight (kg) & $3120.96 \pm 397.02$ & $3207.80 \pm 396.97$ & 1.130 & 0.265 \\
\hline Mother's age (years old) & $29.35 \pm 5.63$ & $28.53 \pm 5.65$ & 0.757 & 0.454 \\
\hline Pregnant time (time) & $2.38 \pm 1.22$ & $2.52 \pm 1.29$ & 0.716 & 0.477 \\
\hline Production time (time) & $1.54 \pm 0.50$ & $1.62 \pm 0.70$ & 0.015 & 0.903 \\
\hline I score (10 points/less than & $30 / 1$ & $184 / 7$ & 1.123 & 0.268 \\
\hline 10 points) & $2.29 \pm 0.73$ & $2.13 \pm 0.69$ & 1.175 & 0.244 \\
\hline Jaundice time (days) & $3.42 \pm 1.14$ & $3.72 \pm 2.14$ & \\
\hline
\end{tabular}

Table 2: Mothers complications comparison of 2 groups in the perinatal period $(x \notin \mathbf{E})$.

\begin{tabular}{|c|c|c|c|c|}
\hline Groups & $\begin{array}{l}\text { Relapse Group After } \\
\text { phototherapy }(n=31)\end{array}$ & $\begin{array}{l}\text { NO Relapse Group After } \\
\text { phototherapy(n=191) }\end{array}$ & $\mathrm{t} / \mathrm{X} 2$ & $\mathbf{P}$ \\
\hline HBsAg positive incidence [n(\%)] & $2(6.45)$ & $18(9.42)$ & 0.286 & 0.593 \\
\hline inflammation gynaecology Incidence [n(\%)] & $4(12.90)$ & $18(9.42)$ & 0.36 & 0.549 \\
\hline respiratory tract infection incidence $[\mathrm{n}(\%)]$ & $0(0.00)$ & $6(3.14)$ & 0.996 & 0.318 \\
\hline TPHA positive incidence [n(\%)] & $0(0.00)$ & $3(1.57)$ & 0.491 & 0.483 \\
\hline HCMV infection rate $[\mathrm{n}(\%)]$ & $1(3.23)$ & $4(2.09)$ & 0.154 & 0.694 \\
\hline HSV-2 infection rate $[\mathrm{n}(\%)]$ & $0(0.00)$ & $3(1.57)$ & 0.491 & 0.483 \\
\hline thyroid dysfunction incidence[n(\%)] & $2(6.45)$ & $10(5.24)$ & 0.077 & 0.782 \\
\hline The incidence of diabetes [n(\%)] & $4(12.90)$ & $19(9.95)$ & 0.250 & 0.617 \\
\hline The incidence of hypertension [n(\%)] & $0(0.00)$ & $10(5.24)$ & 1.692 & 0.193 \\
\hline $\begin{array}{l}\text { Light the Mediterranean anemia incidence } \\
\qquad[\mathrm{n}(\%)]\end{array}$ & $2(6.45)$ & $9(4.71)$ & 0.171 & 0.680 \\
\hline Incidence of uterine fibroids [n(\%) & $0(0.00)$ & $2(1.05)$ & 0.326 & 0.568 \\
\hline
\end{tabular}

\section{Diagnostic Criteria for Neonatal Jaundice}

Diagnostic criteria for neonatal jaundice as the documents published shows [3]. That is total bilirubin level: $\sim 24$ hours $\geq 103 \mu \mathrm{mol} / \mathrm{L}, \sim 48$ hours $\geq 154 \mu \mathrm{mol} / \mathrm{L}, \sim 72$ hours $\geq 205 \mu \mathrm{mol} / \mathrm{L}$, $\sim 72$ hours $\geq 257 \mu \mathrm{mol} / \mathrm{L}$. Total serum bilirubin detection by diazo method and giving priority to with indirect bilirubin.

\section{Diagnostic Criteria for Relapse after Neonatal Jaundice Phototherapy}

Serum total bilirubin level of the neonate is greater than 256 umol/L after they Stop phototherapy $24 \mathrm{~h}$ OR their Serum total bilirubin is still rising after receiving 4-6 hours phototherapy.

\section{Neonatal ABO Hemolytic Disease Diagnostic Criteria}

In neonatal hemolysis three positive (direct anti globulin test, free antibody test and erythrocyte antibody release test), and the mother's blood type is " $\mathrm{O}$ " type, children with blood type for the " $\mathrm{A}$ " or "B", anemia $(\mathrm{Hb}<140 \mathrm{~g} / \mathrm{L})$ shall prevail

\section{Methods}

\section{Phototherapy}

Using the Blue light therapy apparatus (model is XHZ - 90, DAVID company) Continuous irradiation therapy. The main peak wavelength is $425 \sim 475 \mathrm{~nm}$. Preheating Phototherapy box 
before treatment. Let the newborn lie in phototherapy box, when temperature of Phototherapy box is about $30 \mathrm{~L}$. Covering the eyes of the newborn with a black patch so as not to damage their retina. And their Perineum, anus be covered with diaper and the other party of body are exposed. Continuous phototherapy, no more than 3 days.

\section{Experimental Observation Indicators}

Testing Sick newborns' Liver function using Roche. Modular. PPE- automatic biochemical analyzer (Germany Roche) before Phototherapy. Mainly including indicators: AFP (alpha fetoprotein, AFP), Alanine aminotransferase (Alanine aminotransferase, ALT), (Aspartate aminotransferase, AST), (total bilirubin, TBil), TBA, (total bile acid, TBA), G6PD (glucose 6-phosphatedehydrogenase, G6PD). Testing Sick newborns' Blood Routine with sysmex2000i Automatic classification of five blood cell meter (Mr Sen business Japanese company). Mainly including indicators: $\mathrm{Hb}$ (Hemoglobin concentration, Hb), HCT (hematocrit, HCT), MCHC (mean corpuscular hemoglobin contentration, MCHC). At the same time determining the $\mathrm{ABO}$ blood group and Rh blood type of children and their mother with direct agglutination method.

\section{Statistical methods}

Put Data in SPSS22.0 statistical software, Count Data using percentage, Comparison between groups by $\chi £$ test. Measurement data is expressed $x \underline{ \pm} \boldsymbol{\theta}$, Comparison between group by t test, $\mathrm{P}<0.050$.The difference was statistically significant.

\section{Result}

Before phototherapy the $\mathrm{Hb}$ of Relapse Group After phototherapy is $(149.63 \pm 14.71) \mathrm{g} / \mathrm{l}$, obviously lower than that of NO Relapse Group After phototherapy (156.02 \pm 17.90$) \mathrm{g} / \mathrm{l}$. $\mathrm{t}=2.169, \mathrm{P}=0.035$, Neonatal Hemolysis disease incidence rate which is $16.13 \%$ among the Relapse Group After phototherapy is higher than that (it is $4.7 \%$ ) of among the NO Relapse Group After phototherapy. $t=5.857, \mathrm{P}=0.016$ The difference of 2 groups 'other indicators have no statistical significance. Indictors are AFP, AL, AST, TBA, TBI, HCT, MCHC and G6PD (P<0.050). As the Table 3 shows, The TBIL of Relapse Group and No Relapse Group are respectively $(272.28 \pm 42.75) \mathrm{umol} / \mathrm{L}(125.49 \pm 53.94) \mathrm{umol} / \mathrm{L}$ $(\mathrm{t}=17.047, \mathrm{P}<0.001)$ after receiving $(24.38 \pm 3.84)$ hours and $(24.02 \pm 1.40)$ hours phototherapy $(\mathrm{t}=0.525, \mathrm{P}=0.604)$ [4-6].

Table 3: The comparison of experimental observation index of 2 groups before phototherapy ( $x \notin \mathbf{E}$ ).

\begin{tabular}{|c|c|c|c|c|}
\hline Groups & $\begin{array}{l}\text { Relapse Group After } \\
\text { phototherapy(n=31) }\end{array}$ & $\begin{array}{l}\text { NO Relapse Group After } \\
\text { phototherapy(n=191) }\end{array}$ & $t / X 2$ & $\mathbf{P}$ \\
\hline $\mathrm{AFP}(\mathrm{ng} / \mathrm{ml})$ & $607.33 \pm 207.66$ & $614.50 \pm 186.92$ & 0.181 & 0.858 \\
\hline ALT(U/L) & $14.01 \pm 4.86$ & $15.32 \pm 8.85$ & 1.213 & 0.229 \\
\hline AST(U/L) & $64.61 \pm 30.16$ & $57.79 \pm 27.01$ & 1.183 & 0.244 \\
\hline $\mathrm{TBA}(\mu \mathrm{mol} / \mathrm{L})$ & $9.93 \pm 6.03$ & $9.42 \pm 6.61$ & 0.425 & 0.673 \\
\hline $\operatorname{TBIL}(\mu \mathrm{mol} / \mathrm{L})$ & $247.90 \pm 45.73$ & $253.91 \pm 41.61$ & 0.688 & 0.496 \\
\hline HCT(\%) & $45.42 \pm 5.92$ & $49.65 \pm 27.37$ & 1.880 & 0.062 \\
\hline $\mathrm{Hb}(\mathrm{g} / \mathrm{l})$ & $149.63 \pm 14.71$ & $156.02 \pm 17.90$ & 2.169 & 0.035 \\
\hline $\operatorname{MCHC}(\mathrm{g} / \mathrm{l})$ & $323.45 \pm 20.31$ & $327.61 \pm 18.43$ & 1.072 & 0.290 \\
\hline G6PD(U/L) & $2698.25 \pm 1770.92$ & $2787.22 \pm 1392.76$ & 0.267 & 0.791 \\
\hline $\begin{array}{l}\text { ABO Hemolysis disease } \\
\text { incidence }[\mathrm{n}(\%)]\end{array}$ & $5(16.13)$ & $9(4.71)$ & 5.857 & 0.016 \\
\hline
\end{tabular}

\section{Discussion}

Jaundice is very common in the neonatal period. Serum bilirubin level rise can cause damage to liver, cardiac muscle and so on organs. The blood-brain barrier of the newborn develops not well and Bilirubin neurotoxicity. Free bilirubin in the blood may be through the blood brain barrier, causes the bilirubin encephalopathy, that affect children intellectual, listening comprehension, and even life threatening. Phototherapy mechanism is through the 425$475 \mathrm{~nm}$ wavelength light promoting $\eta$ aZ type combined bilirubin isomerization, accelerating the oxidation reaction of light, making it soluble, prompted bilirubin discharge, so that to prevent bilirubin encephalopathy [7]. In clinical practice, however, bilirubin rebound after phototherapy, occurrence probability is generally above $10 \%$ [8], occurrence probability is even $13 \%$. Therefore, the study of factors influencing the bilirubin rebound after phototherapy is of great significance.
The most common cause of Neonatal hemolytic jaundice is ABO hemolyte caused by Maternal-Fetal Blood Group Incompatibility. The most common is that mother's blood type is 0 and fetal blood type is A or B. Its pathogenesis is that fetal paternal genes coding on the red cell membrane antigen entry the matrix in fetal period and Mother IgG antibody is produced. IgG antibody through the placenta into the fetal body and then it forms sensitized erythrocyte. It resulted in autoimmune hemolytic reaction [9that Sensitization erythrocyte destroyed by macrophages and natural killer cells in the body of the newborns .According to reports by Han Xueqin, phototherapy for 331 cases full-term newborn of ABO hemolytic disease is average of 80.1 hours. $8.8 \%$ of children receive blood exchange transfusion and blood transfusion therapy. $19.0 \%$ of children Infusion albumin. Infusion $26.0 \%$ of children with intravenous immunoglobulin. But there is still 1.8\% sick newborn return to hospital because Jaundice rebound after them leaving hospital 4 days. There is a significant difference between the 
cases of two groups of children with $\mathrm{ABO}$ hemolytic disease after phototherapy jaundice rebound in this study $[9,10]$.

The reason is quite likely that: Due to a large number of red blood cell destruction, release a large amount of hemoglobin which resulted in Nonbinding bilirubin content in plasma increased. It is so much that Beyond the liver cells absorb, combination and shedding processing capacity. What's more, both hypoxia caused by $\mathrm{ABO}$ hemolytic disease and toxic substances released due to red blood cell been damaged can weaken the liver cells capability of metabolic bilirubin. Thus Nonbinding bilirubin content retention cause Jaundice is heavy and long duration. Red blood cells and glucose - 6 - phosphate dehydrogenase (G6PD) deficiency is a common hereditary erythrocytic enzyme disease which incidence is high in area south of Yellow River basin in China. In the neonatal period, the onset time of G6PD deficiency within 6 days after birth and often overlaps with the physiological jaundice time. At the same time lack of G6PD cause red blood cells eventually be swallowed by Monocyte-macrophages and reduce liver cell transformation ability of bilirubin And then resulting in high blood bilirubin $[11,12]$. G6PD deficiency infants, although no obvious hemolysis occurred. G6PD deficiency Will delay the fade of high neonatal bilirubin concentration, increase the phototherapy time and hospitalization days [12] reported by JUN Cheng.

In this study, the G6PD numerical of two groups has no statistical difference. It may be thanks to strengthen control of pathogen infection after birth and taboo to take pearl, baby Dan tea, honeysuckle and openings tea. Don't wear contain a smell of camphor ball clothing. Breastfeeding mothers are prohibited from taking antioxidant drugs. Thereby reducing the cause of acute hemolysis induced factors. While this study neonatal hemolytic jaundice patients often have anemia, it is Suggest that low hemoglobin concentration may be the important factors influencing the bilirubin rebound after phototherapy. That is Consistent with Cheng jian's report [13]. Generally because the sick children immunity is low, especially newborn, they cannot produce antibody and vulnerable to infection by Pathogen such as cytomegalovirus, herpes simplex virus, coxsackie virus, virus, rubella virus, hepatitis b virus, hepatitis c virus, parvovirus B19, escherichia coli, staphylococcus aureus, $\mathrm{n} / \mathrm{med}$ tuberculosis bacili, toxoplasma infection, Treponema pallidum. Pathogen infections suppress glucuronyl transferase activity and affect Bilirubin metabolism which resulted in hyperbilirubinemia and delay of time of jaundice subsided.

The TORCH infection mainly cytomegalovirus infection can cause DNA strand break combined with the liver function of the patients did not improve, Cholinesterase activity is low. That can easily lead to the damage to the liver cells and cholestasis, Promote Liver cells enzyme released into the blood stream and affect bile acid metabolism. The difference of ALT, TBA etc. main indicators of liver function of two group's sick newborn in this study had no statistical significance. That is consistent with the report of ChengJian. Because in the early liver disease, jaundice is the basically and only symptoms and other indicators liver function are rarely abnormal. The median onset age of the newborn is about 40 days which is about $5 \%$.

Other reports show that the rise of direct bilirubin level, Cereal third transaminase and aspertate aminotransferase level suggests the possibility of liver disease. When Serum bilirubin level above $20 \mathrm{mu} \mathrm{mol} / \mathrm{L}$ and the ratio of total bilirubin is $>20 \%$, it shows that direct bilirubin is high $[14,15]$. Such cases very little in this study. Thanks to that immunity of Children with high blood bilirubin can depend on maternal antibody, good medical environment and medical personnel protection consciousness is strong, the various pathogens complications greatly reduce. This research also shows that: the difference of alpha fetal protein of the two groups has no statistical significance. Acording to the report [16], although high level alpha fetal protein can detect a lot among children with high blood bilirubin, it is mainly related to premature birth, low birth weight. So this study not includes these kind sick children.

\section{Conclusion}

Above all, full-term neonatal jaundice relapse after phototherapy that is relative to low hemoglobin concentrations and $\mathrm{ABO}$ hemolytic disease. But it has little correlation with it liver function main indicators and G6PD. So using a variety of test indictors in the study of full-term neonatal jaundice relapse after phototherapy, Learn about what is the reason leading obviously relapse after phototherapy so to attract the attention of clinicians. These risk factors should be considered when making follow-up plan after phototherapy. Improving the treatment effect. It is of great significance to ensure the healthy growth of newborn and their family happiness

\section{The limitation of the study}

This study has some limitations,

a) Cases in this study are from our hospital. The research results can only reflect the situation of just local area not other areas.

b) Due to the selection for a long time. The detect time of observation indexes last too long. There is limitation on quality control.

c) Due to unknown complications of the two groups it may reduce the comparability

\section{Acknowledgement}

These project research achievements with the help and support of leaders and colleagues in many aspects. Especially the team member's good effort. Here, we appreciate my colleagues who make great effort to this study.

\section{References}

1. Mutlu M, Cayır Y, Aslan Y (2014) Urinary tract infections in neonates with jaundice in their first two weeks of life. World Journal of Pediatrics 10(2): 164-167.

2. Li Ying Xing, Li Wen Chen, Yin Wu (2015) Blue light combined nursing intervention for neonatal jaundice clinical value analysis. Chinese modern doctor 53(1): 77-79. 
3. Fang Guo Ding (2001) Recommended protocol for intervention in neonatal jaundice. Chinese Journal of practical Pediatrics 16(8):185187.

4. Shao Han Nong, Yan Ming Xie, Yu Hui Yu (2002) The value of albumin application in the phototherapy of jaundice neonates. Guangdong medicine 23(4): 379-381.

5. Qiang Xi, Zhu Wen Yi (2004) Pediatry [M] Version 6. Beijing: people's Health Publishing House, pp. 138-411.

6. Morag I, Okrent AL, Strauss T, Staretz-Chacham O, Kuint J, et al. (2015) Early neonatal morbidities and associated modifiable and nonmodifiable risk factors in a cohort of infants born at 34-35 weeks of gestation. J Matern Fetal Neonatal Med 28(8): 876-882.

7. Smith RW, Mohamed A, Young J (2016) Factors associated with car seat test failure in late preterm infants: A retrospective chart review. Paediatr Child Health 21(1): 16-20.

8. Nakimuli A, Mbalinda SN, Nabirye RC, Kakaire O, Nakubulwa S, et al. (2015) Still births, neonatal deaths and neonatal near miss cases attributable to severe obstetric complications: a prospective cohort study in two referral hospitals in Uganda. BMC Pediatrics 15(1): 44-51.

9. Zhi Feng Gao, Li Hua Hu (2013) Analysis of correlation between blood group antibody titer of 0 type pregnant women and hemolytic disease of newborn. Journal of Clinical Hematology 26(4): 217-221.
10. Xue Qin Han, Jian Jun Tang, Bin Xia (2016) Clinical analysis of abo hemolytic disease associated with hyperbilirubinemia in term neonates. Chinese Journal of Clinical Medicine for Women and Children 12(2): 154-158.

11. Xiao Ming Li, Kai Hui Xing, Tai Ping Zhuang (2016) Comparison of clinical features of hyperbilirubinemia caused by abo hemolytic disease and G6PD deficiency in term neonates. Hainan medicine 27(7): 1060-1062.

12. Jun Chen, Luan Ying Tian, Lu Huang (2012) Effect of g6pd deficiency on the course of hyperbilirubinemia in neonates. Hainan medicine 23(13): $13-14$.

13. Jian Chen, Zhi Yong Jiang, Wei Hong Sun (2014) Factors influencing the effect of phototherapy for jaundice in term neonates. Journal of Guangxi Medical University 31(3): 483-485.

14. Xue Ling Ma (2015) Evaluation of liver function and prognosis of infantile cholestatic liver disease. Hebei Medical University.

15. Dong Ying Zhao, Zhen Juan He, Jian Xing Zhu (2013) The value of serum total bile acid and $\gamma$-glutamyl transpeptidase in evaluating the etiology of delayed jaundice. Journal of Shanghai Jiaotong University 33(7): 931935.

16. Xiao Bing Zhu (2015) Expression of c reactive protein and alpha fetoprotein in neonatal jaundice. Chongqing medicine 4(14): 1968-1970.

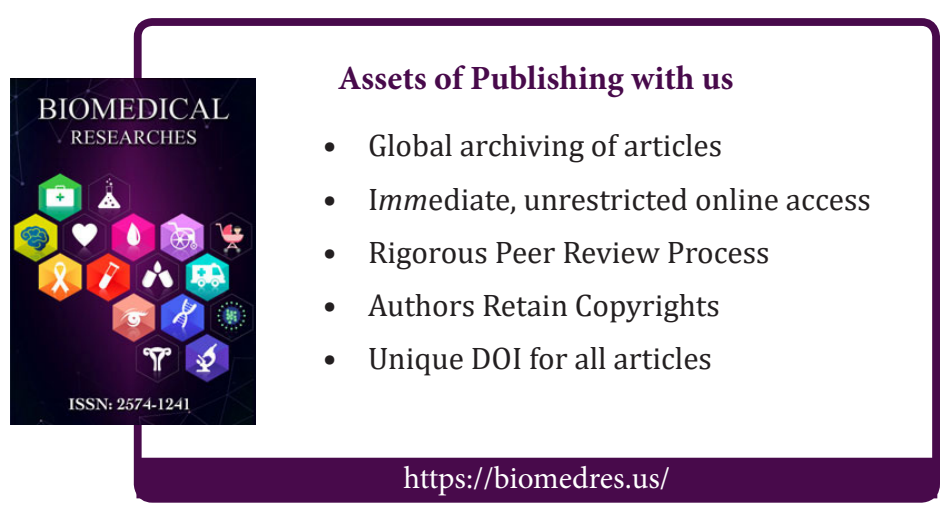

